\title{
Eigenmodes of Nonlinear Dynamics: Definition, Existence, and Embodiment into Legged Robots with Elastic Elements
}

\author{
Dominic Lakatos $^{1}$, Werner Friedl ${ }^{1}$ and Alin Albu-Schäffer ${ }^{1,2}$
}

\begin{abstract}
Pogo-stick bouncing or the spring loaded inverted pendulum represent fundamental dynamics models for hopping and running in legged locomotion. However, these conceptual models are in general of lower order than the elastic multibody dynamics of versatile segmented legs. The question how to embody these simple models into real robot leg designs still has not been completely answered so far. The concept of eigenmodes for linear systems provides a tool to separate high-dimensional, coupled dynamics in one-dimensional (1-D) invariant ones. However, the dynamics of segmented legs is in general nonlinear such that even the existence of periodic motions, as appearing typically in locomotion tasks, cannot be generally guaranteed without changing intrinsic dynamics behavior substantially by control. This paper extends the concept of eigenmodes, which is well-known for linear systems, to the nonlinear case. By proposing a method for selecting the design parameters of multibody systems such that desired eigenmodes are achieved, the problem of embodying fundamental locomotion modes into legged systems is resolved. Examples of practically realizable leg designs are provided, which proof the existence of invariant, 1-D oscillation modes in nonlinear, elastic robot dynamics. An experiment on a multilegged robotic system validates that energetic efficiency can be gained by the proposed approach.
\end{abstract}

\section{INTRODUCTION}

The uses of springs in legged locomotion are known at least since the work of Alexander [1] and lead to the general robotic concept of series elastic actuators (SEA) [2]. The springs moderate impact forces and save energy by a bouncing effect as implemented in fundamental models of hopping and running with a linear elastic leg [3], [4], [5]. Although these models capture the basic principles of legged locomotion, versatile movement in almost any terrain requires the introduction of segmented legs. This leads (in combination with the springs) to nonlinear elastic multibody dynamics of much higher order and complexity than the templates cited above. Fundamental principles of dynamic legged locomotion are based upon cyclic movements which evolve on a lower-dimensional submanifold than the configuration space of the articulated legs. For instance, the motion of Raibert's conceptual hoppers [3] is predominated by the elastic dynamics along a straight line (in parallel to gravitational acceleration) which represents a onedimensional (1-D) submanifold of 3D-space. The hypothesis that the high-dimensional, nonlinear dynamics anchored in a

\footnotetext{
${ }^{1}$ D. Lakatos, W. Friedl and A. Albu-Schäffer are with the Robotic Mechatronic Center (RMC), Institute of Robotics and Mechatronics, German Aerospace Center (DLR), D-82234 Oberpfaffenhofen, Germany dominic.lakatosedlr.de

${ }^{2}$ A. Albu-Schäffer is also with Technical University Munich, Chair of Sensor Based Robots and Intelligent Assistance Systems, Department of Informatics, D-85748 Garching, Germany alin.albu-schaeffer@dlr.de
}

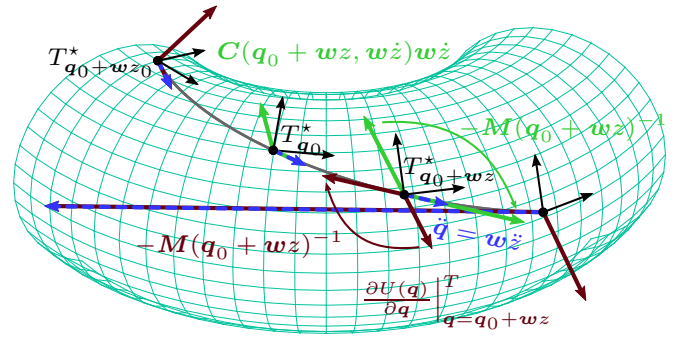

Fig. 1. Geometric representation of modal invariant nonlinear elastic multibody dynamics on the torus manifold of the configuration space. For any displacement $\boldsymbol{q}_{0}+\boldsymbol{w} z$ and velocity $\boldsymbol{w} \dot{z}$ along the mode, the Coriolis/centrifugal forces $\boldsymbol{C}\left(\boldsymbol{q}_{0}+\boldsymbol{w} z, \boldsymbol{w} \dot{z}\right) \boldsymbol{w} \dot{z}$ and potential (gravity and elastic) forces $\left(\partial U(\boldsymbol{q})^{T} / \partial \boldsymbol{q}\right)\left(\boldsymbol{q}_{0}+\boldsymbol{w} z\right)$, cause an acceleration exclusively tangential to the mode, i.e., $\ddot{\boldsymbol{q}}=\boldsymbol{w} \ddot{z}=$ $-\boldsymbol{M}\left(\boldsymbol{q}_{0}+\boldsymbol{w} z\right)^{-1}\left[\boldsymbol{C}\left(\boldsymbol{q}_{0}+\boldsymbol{w} z, \boldsymbol{w} \dot{z}\right) \boldsymbol{w} \dot{z}+\left.\frac{\partial U(\boldsymbol{q})}{\partial \boldsymbol{q}}\right|_{\boldsymbol{q}_{0}+\boldsymbol{w} z} ^{T}\right]$.

complex legged animal collapses to strongly reduced order template dynamics, like the spring loaded inverted pendulum (SLIP) model [4] and extensions [6], [7], [8], [9], is further supported experimentally by biologists [10]. Mathematicians, biologists, roboticists and control theoreticians of [11] agree that embodying these basic oscillation modes into the high order multibody dynamics of articulated legged systems is a key aspect of energy efficient and performant locomotion.

The implementation of reduced order locomotion dynamics into articulated robotic systems has been successfully achieved by methods of feedback control: A pioneering work in this field is the introduction of the concept of virtual model control [12], [13], which has been, e. g., applied to implement SLIP dynamics in an articulated robotic leg in [14]. The method of hybrid zero dynamics has been proposed to design an 1-D submanifold of limit cycle walking and running (with a dynamics diffeomorphic to the SLIP [15]) by control which takes the contact dynamics of articulated legged systems into account [16], [17]. Finally, the concept of operational space control has been adopted to the multilegged case [18], where the number of actuator degrees of freedom generally surpasses the number of task coordinates. This control principle has been further exploited to generate a variety of quadrupedal gaits [19].

Our previous work differs from the above concepts, since it aims at exciting the natural oscillatory dynamics of elastic multibody systems rather than at changing it by control. By exploiting the damping properties inherent in any physical system, we showed that a simple bang-bang like controller 
is able to excite and sustain a limit cycle in compliantly actuated robotic systems [20]. We extended this control principle by an adaptive part which first extracts and then excites the principal components of the natural motionleading to the excitation of an intrinsic mechanical oscillation mode of the system [21]. Following the concept of resonance from linear systems theory, this control approach increases the energy efficiency [22]. However, as the controller excites the natural dynamics, special efforts need to be undertaken in the design of the system, such that its dynamics modes optimally match to most relevant tasks.

Therefore, we focus in this paper on introducing a concept which exploits the degrees of freedom of the mechanism design to embody fundamental oscillation modes of the task dynamics into the plant. By changing the configuration of the elastic multibody system, our recent method [23] adjusts the generalized eigenvectors of the nonlinear inertial and elasticity matrices to locally match the direction of the task. This paper goes one step further and introduces the concept of eigenmodes of nonlinear dynamics, which ensures the modal invariance properties in the entire range of the task space. Thereby, we extend the linear generalized eigenvalue problem intuitively to the nonlinear case and propose an algorithm to embody desired eigenmodes corresponding to the task into the elastic multibody dynamics of, e. g., segmented legs.

\section{IDEA}

The basic goal is to design a locomotor system which is

(i) versatile regarding movement in almost every terrestrial environment;

(ii) mechanically robust against impacts and contact forces occurring in particular for dynamic gaits;

(iii) energetically efficient regarding output power and energy consumption.

The movement versatility requirements (i) can be achieved by introducing segmented legs with sufficient degrees of freedom. Mechanical robustness (ii) can be achieved by having elasticities in the drive train of the joints. Energy efficiency (iii) can be approached if the desired task dynamics fits to the intrinsic dynamical behavior of the plant and if the control excites only this natural dynamics. Fundamental models of legged locomotion which display decoupled task dynamics such as the spring loaded inverted pendulum (SLIP) or the pogo-stick are examples of desired task dynamics. The translational degree of freedom (DoF) of the leg axis ${ }^{1}$ in the SLIP model is dynamically invariant w.r.t. the rotational DoF of the leg angle. In this template model, the translational DoF accounts for energy transformation from kinetic to potential and vice versa and the rotational DoF for redirection of the contact force. The kinetic energy of the flight phase is stored in a linear spring and the resulting elastic force is redirected from the touchdown to the takeoff direction. Therefore, the SLIP model is composed of two dynamical modes: due to the spring, the translational mode

\footnotetext{
${ }^{1}$ The leg axis denotes the directed distance between the hip and the foot.
}

is oscillatory; The rotational motion of the leg is a so-called rigid body mode representing the dynamics of an inverted pendulum. This dynamics, however, essentially deviates from the generally nonlinear and coupled dynamics of segmented legs. The basic idea of this paper is to design the viscoelastic multibody system of the legged robot such that its dynamics encompasses the fundamental template models of locomotion. Embodying the dynamical modes of template dynamics, which are in general of reduced order, into the design of visco-elastic multibody systems is what we call modal dynamics matching.

\section{THEORY OF MODAL DYNAMICS MATCHING}

\section{A. Structural dynamics model}

Consider the dynamics

$$
\boldsymbol{M}(\boldsymbol{q}, \boldsymbol{\zeta}) \ddot{\boldsymbol{q}}+\boldsymbol{b}(\boldsymbol{q}, \dot{\boldsymbol{q}}, \boldsymbol{\zeta})=-\frac{\partial U_{\mathrm{e}}(\boldsymbol{q}, \boldsymbol{\zeta})^{T}}{\partial \boldsymbol{q}}
$$

of which the intrinsic dynamics behavior is to be altered such that it displays invariant oscillation modes. Herein, $\boldsymbol{q} \in \mathbb{R}^{n}$ are configuration variables and $\zeta \in \mathbb{R}^{N}$ are design parameters of the multibody system such as kinematic, inertial, and elastic parameters, respectively. The positive definite $n \times n$ inertia matrix is denoted by $\boldsymbol{M}$ and $U_{\mathrm{e}}$ represents the elastic potential which is a strictly convex function of $\boldsymbol{q}$. The generalized bias force

$$
\boldsymbol{b}(\boldsymbol{q}, \dot{\boldsymbol{q}}, \boldsymbol{\zeta})=\boldsymbol{C}(\boldsymbol{q}, \dot{\boldsymbol{q}}, \boldsymbol{\zeta}) \dot{\boldsymbol{q}}+\frac{\partial U_{\mathrm{g}}(\boldsymbol{q}, \boldsymbol{\zeta})^{T}}{\partial \boldsymbol{q}}
$$

comprises the Coriolis/centrifugal and gravitational effects, respectively.

\section{B. Eigenmodes of linear dynamics}

Consider the linearization of the dynamics (1)

$$
\boldsymbol{M}\left(\boldsymbol{q}_{0}, \boldsymbol{\zeta}\right) \Delta \ddot{\boldsymbol{q}}=-\boldsymbol{K}\left(\boldsymbol{q}_{0}, \boldsymbol{\zeta}\right) \Delta \boldsymbol{q},
$$

where the Hessian of the elastic potential ${ }^{2}$

$$
\boldsymbol{K}\left(\boldsymbol{q}_{0}, \boldsymbol{\zeta}\right)=\left.\frac{\partial^{2} U_{\mathrm{e}}(\boldsymbol{q}, \boldsymbol{\zeta})}{\partial \boldsymbol{q}^{2}}\right|_{\boldsymbol{q}=\boldsymbol{q}_{0}}
$$

is referred to as the stiffness at the equilibrium position $\boldsymbol{q}_{0}$. To solve (3), the complex (harmonic) ansatz $\Delta \boldsymbol{q}(t)=$ $\boldsymbol{w} \hat{z} \exp (\jmath \omega t)$ with constant amplitude $\hat{z}$ and frequency $\omega>0$ gives rise to the generalized eigenvalue problem

$$
\begin{aligned}
\lambda \boldsymbol{M}\left(\boldsymbol{q}_{0}, \boldsymbol{\zeta}\right) \boldsymbol{w} & =\boldsymbol{K}\left(\boldsymbol{q}_{0}, \boldsymbol{\zeta}\right) \boldsymbol{w} \\
\text { s.t. }\|\boldsymbol{w}\|^{2} & =1 .
\end{aligned}
$$

Herein, the eigenvalue $\lambda=\omega^{2}$ denotes the squared eigenfrequency of the oscillation along the eigenvector $\boldsymbol{w}$. In general, (5) has $n$ distinct solutions $\left\{\lambda_{i}, \boldsymbol{w}_{i}\right\}$, for $i=1, \ldots, n$, which are referred to as the eigenmodes of the linear dynamics (3).

The classical generalized eigenvalue problem (5) can be interpreted according to Newton's third law as balance of

\footnotetext{
${ }^{2}$ Strictly speaking, the linearization would include the derivative of the generalized gravitiy force as well, which would add as another "stiffness" component.
} 
inertial and elastic forces, $\lambda \boldsymbol{M} \boldsymbol{w} \hat{z}$ and $\boldsymbol{K} \boldsymbol{w} \hat{z}$, respectively. Any displacement $z \in \mathbb{R}$ along the eigenvector $\boldsymbol{w}, \Delta \boldsymbol{q}=$ $\boldsymbol{w} z$, produces an elastic force which causes an acceleration of the system in the same direction as the displacement, i.e., $\Delta \ddot{\boldsymbol{q}}=-\lambda \Delta \boldsymbol{q}=-\boldsymbol{M}^{-1} \boldsymbol{K} \Delta \boldsymbol{q}$. In particular, the acceleration $\Delta \ddot{\boldsymbol{q}}$ is proportional to the displacement $\Delta \boldsymbol{q}$. As a result, any motion of the system starting in the direction of the eigenvector $\boldsymbol{w}$, i. e., $\Delta \boldsymbol{q}=\boldsymbol{w} z$, evolves strictly along $\boldsymbol{w}$, since the system accelerates exclusively along $\boldsymbol{w}$.

\section{Eigenmodes of nonlinear dynamics}

The physical interpretation of the generalized eigenvalue problem for linear dynamics based on Newton's third law allows us to propose an intuitive extension of the concept of eigenmodes for general nonlinear dynamics (1).

Definition 1: Let $\boldsymbol{q}_{0}$ be an equilibrium position of the system (1) and let $\boldsymbol{w} z$ and $\boldsymbol{w} \dot{z}$ be displacement and velocity of amplitudes $z \in \mathbb{R}$ and $\dot{z} \in \mathbb{R}$, respectively. Then, $\boldsymbol{w}=$ const. is an eigenvector of (1), if there exists an acceleration amplitude $\ddot{z} \in \mathbb{R}$ such that

$$
-\boldsymbol{M}\left(\boldsymbol{q}_{0}+\boldsymbol{w} z\right) \boldsymbol{w} \ddot{z}=\boldsymbol{b}\left(\boldsymbol{q}_{0}+\boldsymbol{w} z, \boldsymbol{w} \dot{z}\right)+\left.\frac{\partial U_{\mathrm{e}}(\boldsymbol{q})}{\partial \boldsymbol{q}}\right|_{\boldsymbol{q}=\boldsymbol{q}_{0}+\boldsymbol{w} z} ^{T}
$$

is satisfied for any $(z, \dot{z}) \in \mathbb{R}^{2}$. A solution $\boldsymbol{w}$ of (7) together with a positive definite metric $S$ such that $\|\boldsymbol{w}\|_{S}^{2}=1$ defines a linear transformation of the form $\Delta \boldsymbol{q}=\boldsymbol{q}-\boldsymbol{q}_{0}=\boldsymbol{w} z$, where $z \in \mathbb{R}$ is referred to as modal coordinate and $\boldsymbol{w}$ as eigenvector of the nonlinear dynamics (1).

A physical interpretation of eigenvectors of nonlinear dynamics can be proposed analogously to the linear case of Sect. III-B: Consider the dynamics (1) with equilibrium position $\boldsymbol{q}_{0}$, where a certain $\boldsymbol{w} z$ with arbitrary length satisfies (7). Assume the system starts at the initial position $\boldsymbol{q}_{0}-\boldsymbol{w} z_{0}$ at rest, i. e., $\dot{\boldsymbol{q}}=\mathbf{0}$. Due to the initial displacement from the equilibrium position $\boldsymbol{q}_{0}$ (along an eigenvector of the system), $-\boldsymbol{w} z_{0}$, an elastic force is produced, which accelerates the system in the direction of the eigenvector. Since the dynamics satisfies (7) for a displacement $\boldsymbol{w} z_{0}$ of arbitrary length and sign, the bias and elastic forces accelerate the system along the eigenvector, at the initial position and at all other positions of the trajectory $\boldsymbol{q}(t)=\boldsymbol{w} z(t)$ and therefore, the system moves tangential to the mode. A geometric representation of such a modal dynamics behavior is given by Fig. 1 .

Definition 2: Let $(\boldsymbol{q}, \dot{\boldsymbol{q}}) \in \mathbb{R}^{2 n}$ be the state of the general nonlinear dynamics (1) with equilibrium position $\boldsymbol{q}_{0}$ and let $\boldsymbol{w}$ be an eigenvector according to Definition 1. Let further $\overline{\boldsymbol{W}}=\operatorname{ker}\left(\boldsymbol{w}^{T}\right)$ be a $n \times(n-1)$ matrix of rank $n-1$ such that $\overline{\boldsymbol{W}}^{T} \boldsymbol{w}=\mathbf{0}$. Then, the set

$$
\mathcal{W}=\left\{(\boldsymbol{q}, \dot{\boldsymbol{q}}) \in \mathbb{R}^{2 n} \mid \overline{\boldsymbol{W}}^{T}\left(\boldsymbol{q}-\boldsymbol{q}_{0}\right)=\mathbf{0}, \overline{\boldsymbol{W}}^{T} \dot{\boldsymbol{q}}=\mathbf{0}\right\}
$$

is said to be an eigenmode of (1).

Note that eigenmodes are invariant sets (cf. [24, Definition 3.9, p. 68]).

Remark 1: The notion of eigenmodes for general nonlinear dynamics strongly differs from the concept of eigenmodes for linear systems. This is as for constant inertia and stiffness matrices, $M$ and $K$, respectively, the corresponding eigenvalue problem (cf. (5)) poses in general $n$ solutions, $\boldsymbol{W}=\left[\boldsymbol{w}_{1}, \ldots, \boldsymbol{w}_{n}\right]$, where $\boldsymbol{W}$ is a nonsingular $n \times n$ matrix which simultaneously diagonalizes $\boldsymbol{M}$ and $\boldsymbol{K}$. Therefore, applying the change of coordinates $\Delta \boldsymbol{q}=\boldsymbol{W} \boldsymbol{z}$ to the corresponding linear dynamics (3) leads to $n$ scalar differential equations which are decoupled in a sense that the $i$-th equation depends only on $z_{i}$ and $\ddot{z}_{i}$. In contrast, solutions $\boldsymbol{w}_{i}$ of the problem (7) exist only for particular nonlinear dynamics (1), where the design parameters $\zeta$ are chosen accordingly. Even if $n$ solutions exist, applying the change of coordinates $\Delta \boldsymbol{q}=\boldsymbol{W} \boldsymbol{z}$ to the dynamics (1) might not lead to $n$ scalar differential equations which are decoupled in a sense described above, despite the corresponding modes are invariant. This will become evident from the examples of Sect. IV-A, IV-B.

\section{Algorithm}

This section derives the methodology which is capable of finding nonlinear mechanical systems with modes matching to the desired dynamics of the task.

Problem 1: Given the desired modes $\boldsymbol{w}_{i}^{\text {des }}$ find parameters $\hat{\zeta}$ satisfying (7) and certain physical and design constraints for which $\boldsymbol{w}_{i}(\hat{\boldsymbol{\zeta}}) \rightarrow \boldsymbol{w}_{i}^{\text {des }}$, for all $i=1, \ldots, n$.

There are two main aspects which make it difficult to solve Problem 1: (i) due to the generally nonlinear transformation $\boldsymbol{x}=\boldsymbol{f}(\boldsymbol{q})$ between configuration and task coordinates, the "shape" of desired eigenvectors $\boldsymbol{w}_{i}^{\text {des }}$ expressed in the tangent space $T_{q}$ of the configuration manifold is not a priori known. (ii) For a general nonlinear dynamics (1), the condition (7) is highly nonlinear in $\boldsymbol{w}_{i} z_{i}$. This suggests to solve the problem in the following steps:

1) Transform the entire problem under a change of coordinates $\boldsymbol{x}=\boldsymbol{f}(\boldsymbol{q})$ to the task-space. Then, the $j$ th row of the $i$-th desired eigenvector $\bar{w}_{j, i}^{\text {des }}=1$ if $j=i$ and zero otherwise. Therefore, the $i$-th desired eigenvector points exclusively in the direction of the $i$-th task-velocity component $\dot{x}_{i}$.

2) Solve the problem

$$
\arg \min _{\boldsymbol{\zeta}_{1}} \sum_{j=1}^{N_{\mathrm{q}}} \sum_{i=1}^{n} \frac{1}{2}\left\|\overline{\boldsymbol{w}}_{i}\left(\boldsymbol{q}_{j}, \boldsymbol{\zeta}_{1}\right)-\overline{\boldsymbol{w}}_{i}^{\mathrm{des}}\right\|^{2}
$$

corresponding to the linearized dynamics (3) (expressed in task-coordinates $\boldsymbol{x}$ ) for a subset $\boldsymbol{\zeta}_{1} \subset \boldsymbol{\zeta}$ of $N_{1}$ design parameters. This problem is subject to a linear eigenvalue problem of the form (5), (6). It is evaluated at relevant configurations $\boldsymbol{q}_{j} \in \mathcal{Q}=$ $\left\{\boldsymbol{q}_{1}, \ldots, \boldsymbol{q}_{N_{\mathrm{q}}}\right\}$ of the task.

3) To remove the remaining coupling terms (omitted due to the linearization), substitute the parameters $\hat{\zeta}_{1}$ solving problem (8) in (5), (6) (expressed in configurationcoordinates $q$ ) to obtain $\boldsymbol{W}$ and transform the original condition (7) according to a change of coordinates $\boldsymbol{q}=\boldsymbol{W} \boldsymbol{z}$. Then, the remaining design parameters $\zeta_{2}$, where $\zeta=\zeta_{1} \cup \zeta_{2}$ can be determined such that remaining coupling terms according to Definition 1 




(a)

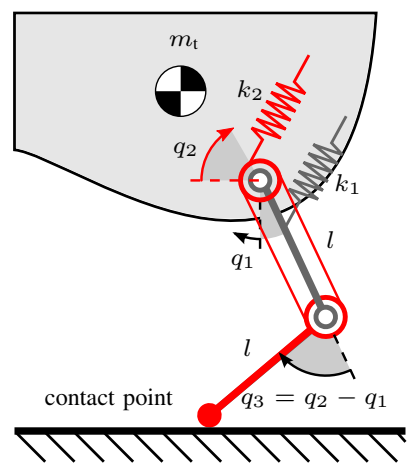

(b)
Fig. 2. (a) Polar coordinates of a SLIP dynamics. (b) Two-segment leg mechanism attached to a trunk with very high inertia.

are removed. This procedure is explained based on the example of Sect. IV-B.

An algorithm to solve step 2) is provided in the appendix.

\section{MOdAL LEG DESIGN}

Fundamental template models of legged locomotion such as the SLIP are based on dynamics which are decoupled in polar coordinates (see, Fig. 2(a)). A translational spring connects the total body mass concentrated at the hip with the pivot point on the ground. This translational degree of freedom will be referred to as the leg axis. The orientation of the leg axis w.r.t. the ground can be (compliantly) actuated under the assumption of very high trunk inertia. The corresponding rotational degree of freedom will be referred to as the leg angle. Note that these task-oriented coordinates can be used to describe all gaits, where the contact between the foot and the ground can be geometrically modeled as a point. Therefore, these fundamental task-oriented coordinates will form the basis for the modal leg design procedure presented in the following.

\section{A. Two-segment leg}

Consider the model of a two-segment leg during stance phase which is assumed to be attached to a body (trunk) with translational degrees of freedoms in the plane (Fig. 2(b)). The thigh is connected to the trunk by a rotational joint with coordinate $q_{1}$. The shank is hinged to the thigh with relative coordinate $q_{3}$. There is a pulley concentric with the hip joint with relative coordinate $q_{2}$ which couples to the knee joint such that $q_{3}=q_{2}-q_{1}$. A point-foot is considered which is constrained to touch the ground such that the configuration of the system is determined by the minimum set of coordinates $\boldsymbol{q}=\left(q_{1}, q_{2}\right)$. Assuming that each leg segment has equal length $l$ and equal mass $m_{1}$ concentrated at the segment center, the inertia matrix of the system takes the form

$$
\boldsymbol{M}(\boldsymbol{q})=\left[\begin{array}{cc}
m_{\mathrm{t}}+\frac{m_{1}}{4} & \left(m_{\mathrm{t}}+\frac{m_{1}}{2}\right) \cos \left(q_{2}-q_{1}\right) \\
\text { sym. } & m_{\mathrm{t}}+\frac{5 m_{1}}{4}
\end{array}\right] l^{2} .
$$

Assuming further that hip joint and pulley are actuated via linear springs with stiffness $k_{1}, k_{2}$, we obtain

$$
\boldsymbol{K}=\left[\begin{array}{cc}
k_{1} & 0 \\
0 & k_{2}
\end{array}\right] \text {. }
$$

Due to our kinematics assumptions, the task-oriented coordinates can be expressed in terms of $\boldsymbol{q}$ as

$$
\boldsymbol{x}=\boldsymbol{f}(\boldsymbol{q})=\left(\frac{\frac{q_{1}+q_{2}}{2}}{l \sqrt{2\left(1+\cos \left(q_{2}-q_{1}\right)\right)}}\right) .
$$

Choosing as design parameters the mass of the legs $m_{1}$ and the ratio of stiffness $k_{2} / k_{1}$, i. e., $\zeta_{1}=\left(m_{1}, k_{2} / k_{1}\right)$, it is found that ideal matching is achieved if $m_{1}=0$ and $k_{2}=k_{1}$, i.e., $\hat{\boldsymbol{\zeta}}_{1}=(0,1)$. Substituting these design parameters in (9) and (10), and using the result to solve the original eigenvalue problem (5) reveals that the eigenvectors ${ }^{3}$

$$
\boldsymbol{W}=\left[\begin{array}{cc}
-1 & 1 \\
1 & 1
\end{array}\right]
$$

of the globally matched dynamics are constant. Note that this is the case although the corresponding dynamics is nonlinear as can be seen by the eigenvalues

$$
\lambda_{1 / 2}=\frac{k_{1}}{m_{\mathrm{t}} l^{2}\left(1 \mp \cos \left(q_{2}-q_{1}\right)\right)}
$$

which clearly depend on the configuration variables $q_{2}-q_{1}$.

Remark 2: The dynamics property of constant eigenvectors (such as in (12)) suggests a linear coordinate transformation of the form $\boldsymbol{z}=\boldsymbol{W}^{-1} \boldsymbol{q}$. The corresponding dynamics expressed in these modal coordinates,

$$
\hat{\boldsymbol{M}}(\boldsymbol{z}) \ddot{\boldsymbol{z}}+{\frac{\partial U_{\mathrm{e}}(\boldsymbol{z})^{T}}{\partial \boldsymbol{z}}}^{T}=-\hat{\boldsymbol{b}}(\boldsymbol{z}, \dot{\boldsymbol{z}})
$$

where

$$
\begin{aligned}
\hat{\boldsymbol{M}}(\boldsymbol{z}) & =\boldsymbol{W}^{T} \boldsymbol{M}(\boldsymbol{q}) \boldsymbol{W} \\
\frac{\partial U_{\mathrm{e}}(\boldsymbol{z})^{T}}{\partial \boldsymbol{z}} & =\left.\boldsymbol{W}^{T} \frac{\partial U_{\mathrm{e}}(\boldsymbol{q})}{\partial \boldsymbol{q}}\right|_{\boldsymbol{q}=\boldsymbol{W} \boldsymbol{z}} ^{T} \\
\hat{\boldsymbol{b}}(\boldsymbol{z}, \dot{\boldsymbol{z}}) & =\boldsymbol{W}^{T}\left(\boldsymbol{C}(\boldsymbol{q}, \dot{\boldsymbol{q}}) \boldsymbol{W} \dot{\boldsymbol{z}}+\frac{\partial U_{\mathrm{g}}(\boldsymbol{q})}{\partial \boldsymbol{q}}\right),
\end{aligned}
$$

is invariant w.r.t. to each mode in a sense that the inertial and elastic dynamics are decoupled. Note that the modal transformation (14)-(17) of the dynamics (1) is exact from a tensorial point of view [25]. In case of matching to SLIPlike dynamics, the bias terms $\hat{\boldsymbol{b}}(\boldsymbol{z}, \dot{\boldsymbol{z}})$ (defined by (17)) which contain the Coriolis/centrifugal and gravity effects constitute structurally the same unidirectional invariance properties as the desired dynamics. As discussed in detail in Remark 3, the motion along the radial mode does not affect the polar mode. Note that the remaining coupling from the polar to the radial mode can be exploited to transport the energy at touchdown to the takeoff via a polar leg motion during the stance phase.

In case of the two-segment leg examples, the modal decoupling properties of Remark 2 can be verified by substituting the ideal design parameters $\hat{\zeta}_{1}$ in (9) and (10) and applying

\footnotetext{
${ }^{3}$ Note that the eigenvectors are not normalized according to (6) to avoid the square root terms in the representation.
} 


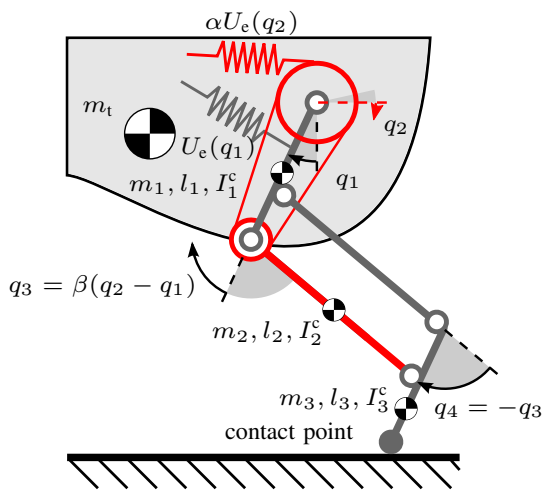

Fig. 3. Three-segment pantograph leg mechanism attached to a trunk with very high inertia.

the modal transformations (15) and (16) on the results, respectively, yielding

$$
\begin{aligned}
\hat{\boldsymbol{M}}\left(\boldsymbol{z}, \hat{\boldsymbol{\zeta}}_{1}\right) & =\left[\begin{array}{cc}
1-\cos \left(2 z_{1}\right) & 0 \\
0 & 1+\cos \left(2 z_{1}\right)
\end{array}\right] 2 m_{\mathrm{t}} l^{2}, \\
\frac{\partial U_{\mathrm{e}}\left(\boldsymbol{z}, \hat{\boldsymbol{\zeta}}_{1}\right)}{\partial \boldsymbol{z}} & =2 k_{1} \boldsymbol{z} .
\end{aligned}
$$

Herein it can be directly seen that the modal inertia as well as the elasticity are decoupled. In particular, (18), (19) reveal that the modal elastic and inertial force are aligned with any displacement along the same mode.

Finally, it should be commented that the requirement of zero leg segment mass is, of course, not exactly implementable. However, due to the kinematic coupling via the pulley, all the actuators including the spring mechanism can be mounted in the trunk which leads to a very light-weight leg design. The mechanism described in the following, allows to avoid this idealized requirement.

\section{B. Three-segment pantograph leg}

Consider the kinematic model of the three-segmented leg which is based on the two-segment leg with an additional segment (representing the foot) hinged to the shank (Fig. 3). The relative angle of this ankle joint is measured between the foot and the shank and is denoted by $q_{4}$. The kinematic constraint $q_{4}=-q_{3}$ keeps the foot segment parallel to the thigh and thereby implements a pantograph mechanism which is known from biomechanics literature [26]. The segment lengths of thigh, shank, foot, are denoted by $l_{1}, l_{2}, l_{3}$ and their inertial parameters (mass, center of mass position, and mass moment of inertia) are labeled as $m_{i}, c_{i}$, and $I_{i}^{\mathrm{c}}$, for $i=$ $1,2,3$, respectively. The center of mass of each segment is assumed at $c_{i}=l_{i} / 2$. To obtain an additional design degree of freedom, the kinematic coupling between the hip and knee is parametrized by $\beta$ such that the corresponding constraint takes the form $q_{3}=\beta\left(q_{2}-q_{1}\right){ }^{4}$ As for the two-segment

\footnotetext{
${ }^{4}$ If the kinematic constraint is implemented by pulleys and cables, then $\beta=r_{\text {hip }} / r_{\text {knee }}$ represents the ratio of radii of the hip and knee pulley, respectively.
}

example, a point contact is assumed which in the treesegment case is located at the tip of the foot. This mechanism has also two kinematic degrees of freedom. The orientations of the thigh and of the pulley concentric to the hip joint relative to the trunk are considered as minimum set of coordinates $\boldsymbol{q}=\left(q_{1}, q_{2}\right)$, respectively. These degrees of freedom are assumed to be actuated via linear springs of which the generalized elastic force are derived from the potential $U_{\mathrm{e}}(\boldsymbol{q})=U_{\mathrm{e}}\left(q_{1}\right)+\alpha U_{\mathrm{e}}\left(q_{2}\right)$, where, e. g., $U_{\mathrm{e}}\left(q_{i}\right)=k_{\text {lin }} q_{i}^{2} / 2+$ $k_{\text {cub }} q_{i}^{4} / 4$, with parameters of the elasticity $\alpha, k_{\text {lin }}, k_{\text {cub }}>0$. The complete set of design parameters comprises: $\boldsymbol{\zeta}=$ $\left(m_{\mathrm{t}}, m_{1}, m_{2}, m_{3}, l_{1}, l_{2}, l_{3}, I_{1}^{\mathrm{c}}, I_{2}^{\mathrm{c}}, I_{3}^{\mathrm{c}}, k_{\text {lin }}, k_{\mathrm{cub}}, \alpha, \beta\right)$.

This example aims at validating two features of modal dynamics matching:

1) The methodology enables to find design solutions which match the desired task dynamics and simultaneously satisfy the implementation constraints such as non-zero link inertia.

2) Design degrees of freedom which are redundant for the eigenvector matching problem (i. e., the non-redundant case satisfies $N_{1}=n(n-1)$ ) can be exploited to achieve modal invariance for terms which are nonlinear even expressed in configuration coordinates $\boldsymbol{q}$ such as gravitational and elastic forces.

On the basis of the latter consideration, a subset of $N_{1}=$ $n(n-1)=2$ design parameters needs to be chosen as optimization variables for the eigenvector matching procedure, while the remaining design parameters need to be fixed. Therefore, a geometric analysis revealed that the parameter assumption $l_{2}=l_{1}+l_{3}$ leads to the task-oriented (polar) coordinates

$$
\boldsymbol{x}=\boldsymbol{f}(\boldsymbol{q})=\left(\begin{array}{c}
\left(1-\frac{\beta}{2}\right) q_{1}+\frac{\beta}{2} q_{2} \\
\left(l_{1}+l_{3}\right) \sqrt{2\left(1+\cos \left(\beta\left(q_{2}-q_{1}\right)\right)\right)}
\end{array}\right),
$$

where the polar angle $x_{1}$ depends linearly on $\boldsymbol{q}$. Furthermore, the link lengths $l_{1}, l_{3}$ can be selected according to workspace considerations. The mass of the trunk $m_{\mathrm{t}}$ will not affect the eigenvectors and can be assumed to be constant. The remaining leg segment parameters $m_{i}, I_{i}^{\mathrm{c}}$, for $i=1,2,3$ influence only the inertial properties and $k_{\text {lin }}, k_{\text {cub }}, \alpha$ only the elastic behavior of the system. Since the matching procedure requires to decouple the inertial as well as the elastic dynamics, one parameter of each category needs to be selected as optimization variables, e. g., $\boldsymbol{\zeta}_{1}=\left(I_{1}^{\mathrm{c}}+I_{3}^{\mathrm{c}}, \alpha\right)$ for our example, while the remaining parameters are considered fixed.

1) The case of linear elasticity: $k_{c u b}=0$ : The application of the procedure of Sect. III-D revealed that constant eigenvectors which ideally match the desired ones can be found for a wide range of the fixed parameters $m_{1}, m_{2}, m_{3}, l_{1}, l_{3}, I_{2}^{\mathrm{c}}, k_{\text {lin }}, \beta$. In particular, it results in implementable values of $I_{1}^{\mathrm{c}}+I_{3}^{\mathrm{c}}>0$ and $\alpha>0$. From inspection of the symbolic expressions ${ }^{5}$ appearing in the eigenvector matching procedure, it can be seen that the family of design

\footnotetext{
${ }^{5}$ This size of problems can still be treated with computer algebra software such as Maple
} 
parameters

$$
\begin{aligned}
I_{1}^{\mathrm{c}}+I_{3}^{\mathrm{c}}= & \frac{3 m_{1}+m_{2}}{4} l_{1}^{2}+\frac{2 m_{1}+m_{2}}{2} l_{1} l_{3} \\
& -\frac{3 m_{2}+m_{3}}{4} l_{3}^{2}+I_{2}^{\mathrm{c}}, \\
\alpha= & \frac{\beta}{2-\beta}
\end{aligned}
$$

leads to globally matching, constant eigenvectors. Substituting the design parameters (20), (21) into the joint space inertia matrix (not explicitly provided) and stiffness $\partial^{2} U_{\mathrm{e}}(\boldsymbol{q}) / \partial \boldsymbol{q}^{2}$ and using the result to solve the corresponding generalized eigenvalue problem, it can be seen that also the eigenvectors

$$
\boldsymbol{W}=\left[\begin{array}{cc}
1 & \frac{\beta}{\beta-2} \\
1 & 1
\end{array}\right]
$$

of the globally matched three-segment leg dynamics are constant. Note that this decoupling property holds even though the leg segment inertial parameters are non-zero. In particular, it can be seen that the generalized elastic force expressed in these modal coordinates (16),

$$
\frac{\partial U_{\mathrm{e}}\left(\boldsymbol{z}, \hat{\boldsymbol{\zeta}}_{1}\right)^{T}}{\partial \boldsymbol{z}}=\left(\begin{array}{c}
\frac{2 k_{\operatorname{lin}}}{2-\beta} z_{1} \\
\frac{2 \beta k_{\operatorname{lin}}}{(2-\beta)^{2}} z_{2}
\end{array}\right),
$$

as well as the modal inertia matrix (not shown here due to space restrictions) are also decoupled.

Remark 3 (Invariance of modal bias terms $\hat{\boldsymbol{b}}$ ):

Inspection of the gravitational bias term $\hat{\boldsymbol{b}}_{\mathrm{g}}(\boldsymbol{z})=\left(\partial U_{\mathrm{g}} / \partial \boldsymbol{z}\right)^{T}$ (cf. (17)) revealed that the parameter family

$$
m_{3}=\left(m_{1} l_{1}+m_{2}\left(l_{1}-l_{3}\right)\right) / l_{3}
$$

leads to the unidirectional invariance property of modal gravity $\hat{b}_{\mathrm{g}, 1}\left(z_{1}=0, z_{2}\right)=0$ for all $z_{2} \in \mathbb{R}$. As a result, any motion of the $z_{2}$-mode does not affect the $z_{1}$-mode via gravitational bias forces as long as $z_{1}=0$.

Since the velocity dependent bias forces $\hat{\boldsymbol{b}}_{\mathrm{C} / \mathrm{c}}(\boldsymbol{z}, \dot{\boldsymbol{z}}$ ) (first term in (17)) are such that the component corresponding to the first mode $\hat{b}_{\mathrm{C} / \mathrm{c}, 1}$ is exclusively bilinear in $\dot{z}_{1}, \dot{z}_{2}$, an analogous conclusion can be drawn for the Coriolis/centrifugal effects: $\hat{b}_{\mathrm{C} / \mathrm{c}, 1}\left(\boldsymbol{z}, \dot{z}_{1}=0, \dot{z}_{2}\right)=0$ for all $\boldsymbol{z} \in \mathbb{R}^{2}$ and $\dot{z}_{2} \in \mathbb{R}$. This property is independent of the parameter condition (24).

Although the eigenvectors of $\boldsymbol{M}(\boldsymbol{q})$ and $\partial^{2} U_{\mathrm{e}}(\boldsymbol{q}) / \partial \boldsymbol{q}^{2}$ are constant for all $\boldsymbol{q} \in \mathbb{R}^{2}$ (cf. (22)), the corresponding eigenvalues,

$$
\begin{aligned}
\lambda_{1} & =\frac{k_{\text {lin }}}{(2-\beta)\left(\Theta_{1}+\Theta_{2} \cos \left(\beta\left(q_{2}-q_{1}\right)\right)\right)}, \\
\lambda_{2} & =\frac{k_{\text {lin }}}{\beta\left(\Theta_{1}-\Theta_{2} \cos \left(\beta\left(q_{2}-q_{1}\right)\right)\right)},
\end{aligned}
$$

where $\Theta_{1}>\Theta_{2}>0$ denote inertia constants, clearly depend on the knee angle $q_{3}=\beta\left(q_{2}-q_{1}\right)$.
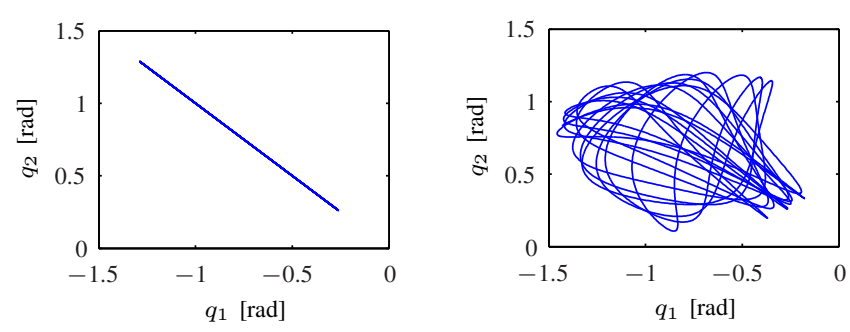

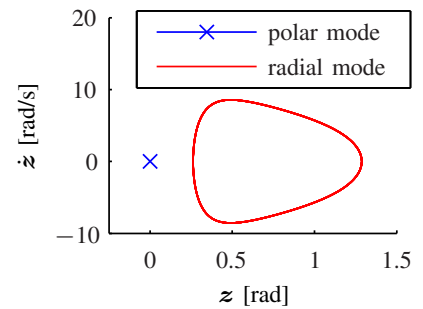

(a) $\alpha=\beta /(2-\beta)$

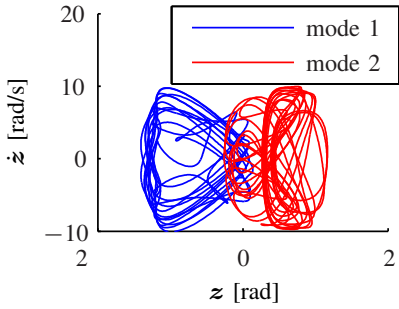

(b) $\alpha=1.5 \beta /(2-\beta)$
Fig. 4. Comparison of the intrinsic dynamics behavior resulting from the modal dynamics matching methodology with a system, where the elastic parameter $\alpha$ is wrongly chosen. It can be seen that only in the former case, the desired invariant dynamics behavior is embodied.

2) The case of nonlinear elasticity: $k_{c u b}>0, \beta=1$ : A particular case of constant eigenvectors of the three segment leg with nonlinear (cubic) elasticities is given if the kinematic coupling parameter $\beta$ is chosen unity, i. e., $\beta=1$. It is found that the elastic force is bidirectionally invariant for an exclusive displacement along one of the modes, i. e.,

$$
\begin{aligned}
& \left.\frac{\partial U_{\mathrm{e}}(\boldsymbol{z})^{T}}{\partial \boldsymbol{z}}\right|_{\forall z_{1} \in \mathbb{R}, z_{2}=0}=\left(\begin{array}{c}
2\left(k_{\operatorname{lin}} z_{1}+k_{\mathrm{cub}} z_{1}^{3}\right) \\
0
\end{array}\right), \\
& \left.\frac{\partial U_{\mathrm{e}}(\boldsymbol{z})^{T}}{\partial \boldsymbol{z}}\right|_{z_{1}=0, \forall z_{2} \in \mathbb{R}}=\left(\begin{array}{c}
0 \\
2\left(k_{\operatorname{lin}} z_{2}+k_{\mathrm{cub}} z_{2}^{3}\right)
\end{array}\right) .
\end{aligned}
$$

Note that this result holds independently of the conditions on the inertial dynamics derived above. It provides evidence for the existence of modally decoupled segmented leg dynamics with nonlinear elasticities as, e. g., implemented by configuration dependent lever arms in biological systems [27].

The three-segment pantograph example validates that using the modal dynamics matching methodology, a nonlinear leg design with globally decoupled modal dynamics can be found which match to a certain task. Thereby, the obtained design parameters are implementable as, e.g., the inertial parameters of the leg segments can be chosen to be nonzero. From biomechanics perspective, this can be an interesting explanation for the prevalence of the pantograph leg kinematics in nature, e. g., for mammals as proposed in [26].

\section{Simulation And EXPERimental RESUlts}

In the following, we check the consequences of the proposed modal leg dynamics regarding invariance of the task relevant oscillation mode, usage for the control of real legged robots and resulting energy efficiency.

\section{A. Invariance properties}

The invariance properties of the leg design with embodied task dynamics are tested and compared with the modally not 


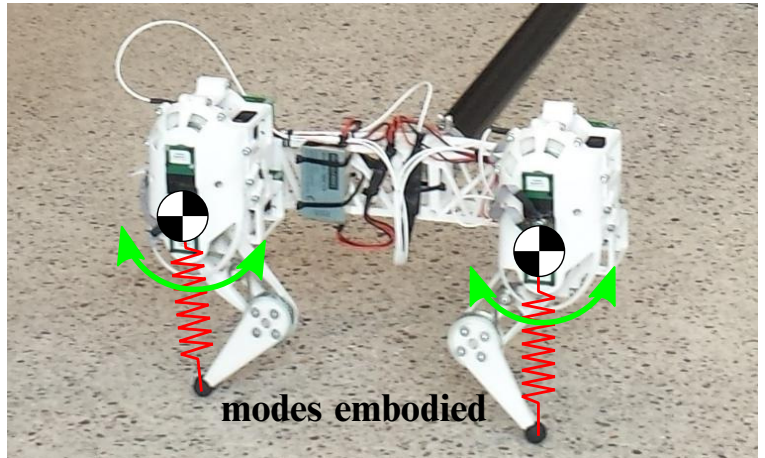

Fig. 5. Compliantly actuated legged robot with task modes embodied used in the experiment.

matching case. Therefore, the natural dynamics of the pantograph leg design (Sect. IV-B) is considered in simulation. The following fixed parameters are used: segment lengths $l_{1}=l_{3}=0.05 \mathrm{~m}$, trunk mass $m_{\mathrm{t}}=0.5 \mathrm{~kg}$, segment masses $m_{1}=0.1 \mathrm{~kg}$ and $m_{2}=0.2 \mathrm{~kg}$, and segment inertia $I_{2}^{\mathrm{c}}=$ $m_{2} / l_{2}^{2} / 12$. The remaining segment length $l_{2}=l_{1}+l_{3}$ results from the kinematic condition required for the linearity of transformation to polar coordinates. The remaining segment inertias $I_{1}^{\mathrm{c}}$ and $I_{3}^{\mathrm{c}}$ are selected according to (20), while $I_{1}^{\mathrm{c}}=$ $I_{3}^{\mathrm{c}}$ is chosen arbitrarily. The segment mass $m_{3}$ results due to the gravity invariance condition (24). Cubic elasticities with $k_{\text {lin }}=1.75 \mathrm{Nm} / \mathrm{rad}$ and $k_{\text {cub }}=0.5 \mathrm{Nm} / \mathrm{rad}^{3}$ are considered and therefore, the kinematic coupling parameter is selected being $\beta=1$. Two cases of the elastic parameter $\alpha$ are compared: the case \#1 of invariant radial dynamics resulting from the matching procedure (cf. (21)), $\alpha=\beta /(2-\beta)$ and the case $\# 2$ where $\alpha$ is chosen wrongly, $\alpha=1.5 \beta /(2-\beta)$. For both cases the full dynamics (1) is simulated for initial deflection $\Delta z_{1}(t=0)=-15$ deg. w. r.t. the equilibrium $z_{1}^{\text {eq. }}=30 \mathrm{deg}$., while $z_{2}(t=0)=0$. Thereby, the relation $\boldsymbol{q}=\boldsymbol{W} \boldsymbol{z}$ defined by the eigenvectors (22) is used. Note that no damping is considered in the model such that possible dynamic coupling effects are not weakened.

In case \#1, the motion of the joints $q_{1}$ vs. $q_{2}$ evolves perfectly along a straight line corresponding to the radial mode of the intrinsic dynamics behavior, as can be seen in Fig. 4(a), upper plot. The projection of the motion in the phase space of the modes $z_{i}$ vs. $\dot{z}_{i}$ (Fig. 4(a), lower plot) shows exclusively a cyclic motion along the $z_{2}$-mode, while $z_{1}$ and $\dot{z}_{1}$ are always zero. The shape of the closed path deviates strongly from the shape of an ellipse which would result in the case of a linear oscillator. Therefore, we may conclude that even in the presence of nonlinear elasticities, Coriolis/centrifugal and gravitational effects, the dynamics of the radial mode is invariant in a sense that once the system reaches the mode it will remain there for all future time. For the case \#2, where the elastic parameter $\alpha$ violates the condition (21) (resulting from the modal dynamics matching algorithm), a fully coupled motion of the joints $q_{1}$ vs. $q_{2}$ results (see, Fig. 4(b)). Note that this simulation result gives rise to the conjecture that the dynamics \#2 is even chaotic.

The comparison shows that only in case of the parameter

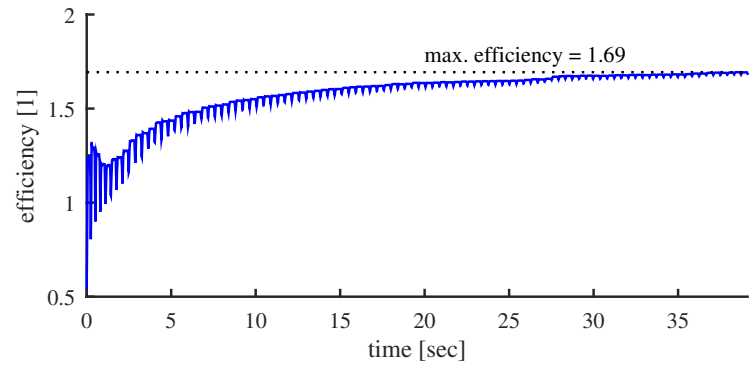

Fig. 6. Efficiency measure defined as the ratio of positive work performed by the joints and motors (cf. (25)). The work of the joints surpasses the work of the motors by a maximum factor of 1.69. This clearly validates the energy efficiency of the modal leg design.

configuration obtained from the modal dynamics matching procedure, the desired invariant system behavior is embodied in the mechanism design. This validates the necessity of the proposed approach.

\section{B. Usage and efficiency of the control system}

The two-segment leg design resulting from the modal dynamics matching procedure described in Sect. IV-A has been implemented as the fore- and hind-leg of a planar quadrupedal robot shown in Fig. 5. The mass of the trunk is about $1.2 \mathrm{~kg}$ and the mass of thigh and shank is about $0.06 \mathrm{~kg}$ and $0.04 \mathrm{~kg}$, respectively. This is in accordance with our assumption of very low leg mass. The two kinematic degrees of freedom of each leg are elastically actuated via springs corresponding to the potential function $U_{\mathrm{e}}=$ $1 / 2 k_{1}\left(q_{1}-\theta_{1}\right)^{2}+1 / 2 k_{2}\left(q_{2}-\theta_{2}\right)$, with spring constants $k_{1}=k_{2}=2 \mathrm{Nm} / \mathrm{rad}$. The motor positions $\boldsymbol{\theta}$ are controlled independently in each servo-unit and can be considered as control input of the system.

Our previously presented jumping control approach [28] has been applied simultaneously to each of the legs. Thereby, a limit cylce excitation is achieved by our previously proposed switching based controller [29] which is used to inject energy along the radial mode. To control the forward hopping speed, a repetitive low-gain method (also described in [28]), which acts along the polar mode, has been considered. An experiment, where the robot has been controlled for hopping at low forward speed, has been conducted. Note that a video showing this experiment is attached to the paper.

On the basis of the measured motor velocity $\dot{\theta}$, joint velocity $\dot{\boldsymbol{q}}$ and elastic torque $\boldsymbol{\tau}=-\partial U_{\mathrm{e}}(\boldsymbol{q}, \boldsymbol{\theta}) / \partial \boldsymbol{q}$, the positive work performed by the joints and motors have been compared considering the efficiency measure

$$
\eta=\frac{\int_{t}^{t+T} \max \left(P_{\mathrm{j}}(s), 0\right) \mathrm{d} s}{\int_{t}^{t+T} \max \left(P_{\mathrm{m}}(s), 0\right) \mathrm{d} s},
$$

where the power of the joints and motors are denoted by $P_{\mathrm{j}}=\dot{\boldsymbol{q}}^{T} \boldsymbol{\tau}$ and $P_{\mathrm{m}}=\dot{\boldsymbol{\theta}}^{T} \boldsymbol{\tau}$, respectively. As can be seen in Fig. 6 , the positive work performed by the joints surpasses the positive work of the motors by a maximum factor of 1.69 . This clearly validates the energy efficiency of the modal leg design. 


\section{CONCLUSION}

The main insight of the paper is that it is possible to design elastically coupled nonlinear systems such that they intrinsically display at large a modal behavior with constant modal vectors. If the initial conditions are along such an eigenvector, the system will continue to move along this direction, being perturbed, if any, only by Coriolis and centrifugal forces. We presented a constructive approach for designing such systems and applied it for the design and analysis of planar segmented legs. An interesting finding is that, for a two-segment leg, constant modes can be obtained only in the limit case of zero leg inertia, which is consistent with the common wisdom of designing the leg segments as lightweight as possible. In contrast, a pantograph leg can display constant modal direction for a large range of realistic parameters. An important result is that the corresponding dynamics consists of a two-dimensional, invariant submanifold of its state-space and thereby validates the existence of periodic motions in multi-degree of freedom nonlinear systems. As demonstrated by the experiments, controlling limit cycles of such a leg is an easy task, leading to energy saving by elastic energy storage.

\section{APPENDIX}

The problem (8) can be solved by applying Newton's method for nonlinear algebraic equations. Thereby, an iteration step $\Delta \zeta_{1}$ is given by the solution of the system of linear equations

$$
\begin{gathered}
\sum_{j=1}^{N_{\mathrm{q}}} \sum_{i=1}^{n} \boldsymbol{H}_{i}\left(\boldsymbol{w}_{i}\left(\boldsymbol{q}_{j}, \boldsymbol{\zeta}_{1}\right)\right)\left(\boldsymbol{w}_{i}\left(\boldsymbol{q}_{j}, \boldsymbol{\zeta}_{1}\right)-\boldsymbol{w}_{i}^{\mathrm{des}}\right) \\
=\frac{1}{\gamma} \sum_{j=1}^{N_{\mathrm{q}}} \sum_{i=1}^{n} \boldsymbol{H}_{i}\left(\boldsymbol{w}_{i}\left(\boldsymbol{q}_{j}, \boldsymbol{\zeta}_{1}\right)\right) \boldsymbol{G}_{i}\left(\boldsymbol{q}_{j}, \boldsymbol{\zeta}_{1}\right) \Delta \boldsymbol{\zeta}_{1}
\end{gathered}
$$

where $\gamma>0$ controls the step length. Due to the normalization condition (6), the Jacobian matrices $\boldsymbol{G}_{i}\left(\boldsymbol{q}_{j}, \boldsymbol{\zeta}_{1}\right)=\partial \boldsymbol{w}_{i}\left(\boldsymbol{q}_{j}, \boldsymbol{\zeta}_{1}\right) / \partial \boldsymbol{\zeta}_{1}$ are rank deficient, i.e., $\operatorname{rank}\left(\boldsymbol{G}_{i}\left(\boldsymbol{q}_{j}, \boldsymbol{\zeta}_{1}\right)\right)=n-1$, even in cases $n \leq N_{1}$. This problem of rank deficiency is solved by transforming the components of the eigenvectors to coordinates $\boldsymbol{y}_{i}=\boldsymbol{y}\left(\boldsymbol{w}_{i}\right) \in$ $\mathbb{R}^{n-1}$ representing the direction of the eigenvector $\boldsymbol{w}_{i}$. The corresponding Jacobian matrix can be expressed as $\boldsymbol{H}\left(\boldsymbol{w}_{i}\right)=\partial \boldsymbol{y}(\boldsymbol{w}) /\left.\partial \boldsymbol{w}\right|_{\boldsymbol{w}=\boldsymbol{w}_{i}}$.

\section{REFERENCES}

[1] R. M. Alexander, "Three uses for springs in legged locomotion," Int. J. of Robotics Research, vol. 9, no. 2, pp. 53-61, 1990.

[2] G. A. Pratt and M. M. Williamson, "Series elastic actuators," in IEEE/RSJ Int. Conf. on Intelligent Robots and Systems, 1995, pp. 399406.

[3] M. H. Raibert, Legged Robots That Balance. The MIT Press, 1986.

[4] R. Blickhan, "The spring-mass model for running and hopping," $J$. of Biomechanics, vol. 22, pp. 1217-1227, 1989.

[5] R. Blickhan and R. Full, "Similarity in multilegged locomotion: bouncing like a monopode," J. of Comparative Physiology A, vol. 173, no. 5, pp. 509-517, 1993.

[6] A. Seyfarth, H. Geyer, M. Günther, and R. Blickhan, "A movement criterion for running." J. of Biomechanics, vol. 35, no. 5, pp. 649-655, 2002.

[7] H. Geyer, A. Seyfarth, and R. Blickhan, "Compliant leg behavior explains basic dynamics of walking and running," Proc. of the Royal Society B, vol. 273, pp. 2861-2867, Nov. 2006.
[8] H.-M. Maus, J. Rummel, and A. Seyfarth, "Stable upright walking and running using a simple pendulum based control scheme," in Advances in Mobile Robotics: Proc. 11th Int. Conf. Climbing and Walking Robots and the Support Technologies for Mobile Machines, 2008, pp. 623-629.

[9] J. Rummel, Y. Blum, H. M. Maus, C. Rode, and A. Seyfarth, "Stable and robust walking with compliant legs," in Proc. of IEEE Int. Conf. on Robotics and Automation, 2010, pp. 5250-5255.

[10] R. Full and D. Koditschek, "Templates and anchors: neuromechanical hypotheses of legged locomotion on land," J. of Experimental Biology, vol. 202, no. 15 , pp. 3325-3332, 1999.

[11] P. Holmes, R. Full, D. Koditschek, and J. Guckenheimer, "The dynamics of legged locomotion: Models, analyses and challenges," SIAM Review, vol. 48, no. 2, pp. 207-304, 2006.

[12] J. Pratt, P. Dilworth, and G. Pratt, "Virtual model control of a bipedal walking robot," in IEEE Int. Conf. on Robotics and Automation, 1997, pp. 193-198.

[13] J. Pratt and G. Pratt, "Intuitive control of a planar bipedal walking robot," in IEEE Int. Conf. on Robotics and Automation, 1998, pp. 2014-2021.

[14] M. Hutter, D. Remy, M. A. Höpflinger, and R. Siegwart, "Slip running with an articulated robotic leg," in IEEE/RSJ Int. Conf. on Intelligent Robots and Systems, 2010, pp. 4934-4939.

[15] I. Poulakakis and J. W. Grizzle, "The spring loaded inverted pendulum as the hybrid zero dynamics of an asymmetric hopper," IEEE Transactions on Automatic Control, vol. 54, no. 8, pp. 1779-1793, 2009.

[16] E. R. Westervelt, J. W. Grizzle, C. Chevallereau, J. H. Choi, and B. Morris, Feedback Control of Dynamic Bipedal Robot Locomotion. CRC Press, 2007.

[17] K. Sreenath, H.-W. Park, and J. W. Grizzle, "Embedding active force control within the compliant hybrid zero dynamics to achieve stable, fast running on MABEL," The Int. J. of Robotics Research, vol. 32, pp. $324-345,2013$.

[18] M. Hutter, H. Sommer, C. Gehring, M. Hoepflinger, M. Bloesch, and R. Siegwart, "Quadrupedal locomotion using hierarchical operational space control," The Int. J. of Robotics Research, vol. 33, pp. 1047$1062,2014$.

[19] C. Gehring, S. Coros, M. Hutter, M. Bloesch, M. Hoepflinger, and R. Siegwart, "Control of dynamic gaits for a quadrupedal robot," in IEEE Int. Conf. on Robotics and Automation, May 2013, pp. 32873292.

[20] D. Lakatos, F. Petit, and A. Albu-Schäffer, "Nonlinear oscillations for cyclic movements in human and robotic arms," IEEE Transactions on Robotics, vol. 30, no. 4, pp. 865-879, 2014.

[21] D. Lakatos, M. Görner, F. Petit, A. Dietrich, and A. Albu-Schäffer, "A modally adaptive control for multi-contact cyclic motions in compliantly actuated robotic systems," in IEEE/RSJ Int. Conf. on Intelligent Robots and Systems, 2013, pp. 5388-5395.

[22] P. Stratmann, D. Lakatos, M. C. Özparpucu, and A. Albu-Schäffer, "Legged elastic multibody systems: Adjusting limit cycles to closeto-optimal energy efficiency," IEEE Robotics and Automation Letters, vol. 2, no. 2, pp. 436-443, 2017.

[23] D. Lakatos and A. Albu-Schäffer, "Modal matching: An approach to natural compliant jumping control," IEEE Robotics and Automation Letters, vol. 1, no. 1, pp. 274-281, 2016.

[24] J.-J. Slotine and W. Li, Applied Nonlinear Control. Prentice-Hall, 1991.

[25] D. Lovelock and H. Rund, Tensors, differential forms, and variational principles. Courier Dover Publications, 1989.

[26] H. Witte, R. Hackert, W. Ilg, J. Biltzinger, N. Schilling, F. Biedermann, M. Jergas, H. Preuschoft, M. Fischer, et al., "Quadrupedal mammals as paragons for walking machines," in Proc. of the Int. Symp. on Adaptive Motion in Animals and Machines (AMAM), 2000, pp. ThA-1-1.

[27] W. Herzog and L. Read, "Lines of action and moment arms of the major force-carrying structures crossing the human knee joint." $J$. of Anatomy, vol. 182, pp. 213-230, 1993.

[28] D. Lakatos, D. Seidel, W. Friedl, and A. Albu-Schäffer, "Targeted jumping of compliantly actuated hoppers based on discrete planning and switching control," in IEEE/RSJ Int. Conf. on Intelligent Robots and Systems, 2015, pp. 5802-5808.

[29] D. Lakatos and A. Albu-Schäffer, "Switching based limit cycle control for compliantly actuated second-order systems," in Proc. of the 19th IFAC World Congress, 2014, pp. 6392-6399. 